\title{
SNR Estimation for FM-DCSK System over Multipath Rayleigh Fading Channels
}

\author{
Guofa Cai*, Lin Wang*, Long Kong ${ }^{+}$, Georges Kaddoum ${ }^{+}$ \\ $*$ Dept. of Communication Engineering, Xiamen University, Fujian 361005, China \\ +University of Québec, École de Technologie Supérieure, Montréal, Canada \\ Email: caiguofa2006@126.com, wanglin@xmu.edu.cn, long.kong.1@ens.etsmtl.ca, georges.kaddoum@etsmtl.ca
}

\begin{abstract}
In this paper, we deal with the problem of maximum likelihood (ML) estimation of the signal-to-noise ratio (SNR) parameter for frequency modulated differential chaos shift key (FM-DCSK) system over multipath Rayleigh fading channels. The ML estimators are derived for various scenarios including data-aided (DA), non-data aided (NDA) and joint DA-NDA estimation by using both the data and pilot symbols. For comparison purposes, the Cramér-Rao lower bounds (CRLBs) for the SNR estimators are derived. The performance of the estimators is evaluated by simulations and comparing with CRLBs in terms of the mean-square-error. Simulated results show that for a large spreading factor the proposed scheme performs well over a wide SNR range in comparisons with CRLBs.
\end{abstract}

Index Terms-FM-DCSK; SNR estimation; Cramér-Rao lower bound (CRLB); multipath Rayleigh fading channels.

\section{INTRODUCTION}

$\mathbf{F}$ REQUENCY modulated differential chaos shift key (FMDCSK) system offers excellent performance under multipath fading or time-varying channels without requiring channel estimation [1-2], which has been used in ultra-wideband (UWB) systems for short-distance communications [3-4]. So far, many researchers have studied the performance of the combination of advanced technology, e.g. cooperative communications [5-6], channel coding [7-8], and multi-input multioutput (MIMO)[9-10], etc., with FM-DCSK modulation. To optimize these schemes, the receiver needs to rely on the estimates of signal-to-noise ratio (SNR). For instance, in a coded FM-DCSK system, the SNR estimation is used to make soft-decision decoding and adjust adaptive strategies; in a DCSK cooperative system, the selection of relay nodes and adopting amplify and-forward (AF) or decode-and-forward (DF) mechanisms are based on the estimates of SNR; in a MIMO-DCSK system, the power control algorithms are also dependent on the estimates of SNR.

For traditional modulations, including phase shift keying (PSK)[11], quadrature amplitude modulation (QAM)[12-13], and frequency shift keying (FSK)[14-15], much work has been done to show the SNR estimation. The maximum likelihood (ML) based estimator, which has asymptotic properties of being unbiased, achieving to Cramér-Rao lower bound (CRLB), is overwhelmingly the most popular approach to obtaining practical estimators (see Chapter 7 of [16]). The CRLB (see Chapter 3 of [16]) gives the minimum variance of unbiased estimators, which is a very useful tool for evaluating the performance of an estimator.
In this work, we derive ML SNR estimators, which include data-aided (DA), non-data aided (NDA) and joint DA-NDA estimators, for FM-DCSK system in multipath Rayleigh fading channels. The CRLBs for ML SNR estimators are derived as a fundamental benchmark that reflects the best achievable performance.

\section{SYSTEM MODEL}

Consider a FM-DCSK communication system, shown in Fig.1. In the FM-DCSK modulator, every bit to be transmitted is represented by two sample functions. For bit "1", the same FM chaotic signal is transmitted twice in succession while bit " 0 " is sent by transmitting the reference chaotic signal followed by an inverted copy of the same signal.

The logistic map is employed as chaotic generator: $x_{k+1}=$ $1-2 x_{k}^{2}$. The chaotic signal is modulated by using FM modulator to generate FM chaotic signal. The FM chaotic signal $z$, after sampling, obtains $2 \beta$ discrete points, where $2 \beta$ is defined as a spreading factor. The $k$-th transmitted signal is represented by a sequence of samples of the chaotic signal, in which the $j$-th sample is given by

$$
s_{j, k}=\left\{\begin{array}{l}
z_{j}, j=2(k-1) \beta+1, \ldots, 2(k-1) \beta+\beta \\
c_{m k} z_{j-\beta}, j=(2 k-1) \beta+1, \ldots, 2 k \beta
\end{array},\right.
$$

where $c_{m k} \in\{-1,+1\}$.

The general channel model of spread spectrum wireless communication systems [2][17-19] is used. The discrete time impulse response of the channel is given by

$$
h(n)=\sum_{l=1}^{L} \alpha_{l} \delta\left(n-\tau_{l}\right),
$$

where $L$ is the number of paths, $\alpha_{l}$ and $\tau_{l}$ are the channel coefficient and the delay of the $l$-th path respectively. Here, we assume that $\alpha_{l}$ are the circularly symmetric complex independent random variables with a Rayleigh distribution.

In most applications [2][17][18], the large spreading factors are generally chosen, thus, the largest multipath time delay is shorter than the bit duration, i.e. $0<\tau_{\text {lmax }} \ll 2 \beta$. In this case, the inter-symbol interference (ISI) is negligible. Hence, similarly to [2][17][18], at the receiver, the decision variable is approximated by

$r_{k} \approx \sum_{j=1}^{\beta}\left(\sum_{l=1}^{L} \alpha_{l} z_{j-\tau_{l}}+n_{j}\right) \cdot\left(c_{m k} \sum_{l=1}^{L} \alpha_{l} z_{j-\tau_{l}}+n_{j+\beta}\right)^{*}$, 


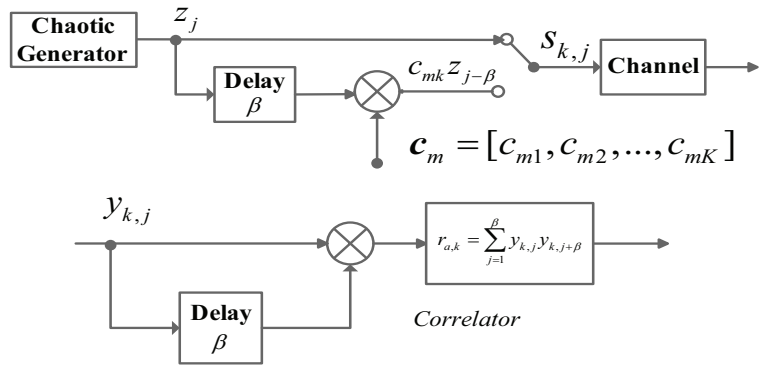

Fig. 1. System model of the FM-DCSK communication system.

where $n$ is a wideband AWGN, characterized by a circularly symmetric complex Gaussian density with zero mean and variance $N_{0}$ (variance $N_{0} / 2$ in the real and imaginary components), and $*$ denotes conjugation operator. In free ISI case, the accurate distribution of Eq. (3) can be calculated by using the method of [20], but the computational complexity is very high owing to using the residue calculus. In [2][5-6][910][17-18][20], it was shown that for large spreading factors, Eq. (3) could be considered as the Gaussian distribution. For a large spreading factor, the following approximated expression is used [2][17-18]:

$$
\sum_{j=1}^{\beta} z_{j-\tau_{l}} z_{j-\tau_{i}} \approx 0, l \neq i
$$

Hence, Eq. (3) is further approximated by

$$
\begin{aligned}
r_{k} \approx & \sum_{j=1}^{\beta}\left(c_{m k} \sum_{l=1}^{L}\left|\alpha_{l}\right|^{2}\left|z_{j-\tau_{l}}\right|^{2}+\sum_{l=1}^{L} \alpha_{l} z_{j-\tau_{l}} n_{j+\beta}^{*}\right. \\
& \left.+c_{m k} \sum_{l=1}^{L} \alpha_{l}^{*} z_{j-\tau_{l}}^{*} n_{j}+n_{j} n_{j+\beta}^{*}\right) .
\end{aligned}
$$

Actually, the effect of multipath fading leads to the loss of partial energy, however, by means of the above approximation we may consider as collecting all the energy. Hence, it is not difficult to derive the mean and variance of the real part $r_{a k}$, respectively, which are given by

$$
\left\{\begin{array}{l}
E\left[r_{a k}\right]=c_{m k} \sum_{l=1}^{L}\left|\alpha_{l}\right|^{2} \frac{E_{b}}{2}, \\
\operatorname{Var}\left[r_{a k}\right]=\sum_{l=1}^{L}\left|\alpha_{l}\right|^{2} \frac{E_{b} N_{0}}{2}+\beta \frac{N_{0}^{2}}{2},
\end{array}\right.
$$

where $E_{b}=\sum_{j=1}^{2 \beta}\left|z_{j}\right|^{2}$ is energy per bit, E[.] denotes the expectation operator and $\operatorname{Var}[$.$] is the variance operator. For$ convenience, we define $h=\sum_{l=1}^{L}\left|\alpha_{l}\right|^{2}$ as the power factor and the transmitted power is supposed to be normalized to one, i.e. $E_{b}=1$. Using the multiple observations $\left\{r_{a k}\right\}_{k=1, \ldots, K}$, the true SNR, $\gamma$, that we wish to estimate, is define as $\gamma=h / N_{0}$. It is mathematically more convenient to use the parameter vector, $\boldsymbol{\theta}=\left[\begin{array}{ll}h & N_{0}\end{array}\right]$, and the function, $g(\boldsymbol{\theta})=h / N_{0}$

\section{SNR ESTIMATION}

Since the channel is slowly fading, we assume that the channel coefficients are constant during the transmission time of a FM-DCSK sequence length $K$. Consider $g$ pilot symbols and $l$ data symbols, so that the total packet is of the length $K=g+l$. The decision vector is defined as $\boldsymbol{r}_{a}=\left[r_{a 1}, r_{a 2}, \ldots, r_{a k}, \ldots, r_{a K}\right]^{T}$, where $[.]^{T}$ denotes the transpose. The information symbol vector is defined by $\boldsymbol{c}_{m}=\left[c_{m 1}, c_{m 2}, \ldots, c_{m k}, \ldots, c_{m K}\right]^{T}$.

\section{A. Data Aided (DA) Estimation Using Pilot Symbols}

For this case, the probability density function (PDF) of $r_{a k}$ is written as

$$
p\left(r_{a k} \mid c_{m k}, \boldsymbol{\theta}\right)=\frac{\exp \left[-\frac{\left(r_{a k}-c_{m k} h / 2\right)^{2}}{\beta N_{0}^{2}+h N_{0}}\right]}{\sqrt{\pi\left(\beta N_{0}^{2}+h N_{0}\right)}} .
$$

With independent received symbols, the joint PDF of the $g$ received symbols is given by

$$
p\left(\boldsymbol{r}_{a} \mid \boldsymbol{c}_{m}, \boldsymbol{\theta}\right)=\frac{\exp \left[-\frac{\sum_{k=1}^{g}\left(r_{a k}-c_{m k} h / 2\right)^{2}}{\beta N_{0}^{2}+h N_{0}}\right]}{\left[\sqrt{\pi\left(\beta N_{0}^{2}+h N_{0}\right)}\right]^{g}} .
$$

Thus, the log-likelihood function (LLF) for the $g$ received symbols is given as,

$\Lambda\left(\boldsymbol{r}_{a} ; \boldsymbol{\theta}\right)=-\frac{g}{2} \ln \pi-\frac{g}{2} \ln \left(\beta N_{0}^{2}+h N_{0}\right)-\frac{\sum_{k=1}^{g}\left(r_{a k}-\frac{c_{m k} h}{2}\right)^{2}}{\beta N_{0}^{2}+h N_{0}}$.

In order to maximize (10), by taking the first-order partial derivative of (10) with respect to $h$ and $N_{0}$, the results are given by

$\frac{\partial \Lambda}{\partial h}=\frac{-\frac{g}{2} N_{0}-\sum_{k=1}^{g} c_{m k}\left(r_{a k}-\frac{c_{m k} h}{2}\right)}{\beta N_{0}^{2}+h N_{0}}+\frac{N_{0} \sum_{k=1}^{g}\left(r_{a k}-\frac{c_{m k} h}{2}\right)^{2}}{\left(\beta N_{0}^{2}+h N_{0}\right)^{2}}$,

$$
\frac{\partial \Lambda}{\partial N_{0}}=-\frac{\frac{g}{2}\left(2 \beta N_{0}+h\right)}{\beta N_{0}^{2}+h N_{0}}+\frac{\left(2 \beta N_{0}+h\right) \sum_{k=1}^{g}\left(r_{a k}-\frac{c_{m k} h}{2}\right)^{2}}{\left(\beta N_{0}^{2}+h N_{0}\right)^{2}} .
$$

Setting the above equations to zero, one obtains the following results

$$
\begin{aligned}
& \sum_{k=1}^{g} c_{m k}\left(r_{a k}-c_{m k} h / 2\right)=0, \\
& \frac{g}{2}\left(\beta N_{0}^{2}+h N_{0}\right)=\sum_{k=1}^{g}\left(r_{a k}-c_{m k} h / 2\right)^{2} .
\end{aligned}
$$

Using (13), the estimate of $h$ is given as

$$
\widehat{h}=\frac{2 \sum_{k=1}^{g} c_{m k} r_{a k}}{g} .
$$

Using (14) and (15), the estimate of $N_{0}$ is given by

$$
\widehat{N}_{0}=\frac{-\frac{\widehat{h}}{2}+\sqrt{\left(\frac{\widehat{h}}{2}\right)^{2}+\frac{2 \beta}{g} \sum_{k=1}^{g}\left(r_{a k}-\frac{c_{m k} \widehat{h}}{2}\right)^{2}}}{\beta} .
$$

Thus, using (15) and (16), the ML estimation of the SNR is found, which is given by $\widehat{\gamma}=\widehat{h} / \widehat{N}_{0}$. 


\section{B. Non-Data Aided (NDA) Estimation Using Data Symbols}

For this case, the PDF of $r_{a k}$ is written as

$$
\begin{aligned}
p\left(r_{a k} \mid \boldsymbol{\theta}\right) & =\frac{\exp \left[-\frac{\left(r_{a k}-h / 2\right)^{2}}{\beta N_{0}^{2}+h N_{0}}\right]+\exp \left[-\frac{\left(r_{a k}+h / 2\right)^{2}}{\beta N_{0}^{2}+h N_{0}}\right]}{2 \sqrt{\pi\left(\beta N_{0}^{2}+h N_{0}\right)}} \\
& =\frac{\exp \left(-\frac{r_{a k}^{2}+\frac{h^{2}}{4}}{\beta N_{0}^{2}+h N_{0}}\right)}{\sqrt{\pi\left(\beta N_{0}^{2}+h N_{0}\right)}} \cosh \left(\frac{h r_{a k}}{\beta N_{0}^{2}+h N_{0}}\right),
\end{aligned}
$$

where $\exp (x)+\exp (-x)=2 \cosh (x)$ is used.

With independent received symbols, the joint PDF of the $l$ received symbols is given by

$$
p\left(\boldsymbol{r}_{a} \mid \boldsymbol{\theta}\right)=\frac{\prod_{k=1}^{l} \exp \left(-\frac{r_{a k}^{2}+\frac{h^{2}}{4}}{\beta N_{0}^{2}+h N_{0}}\right) \cosh \left(\frac{h r_{a k}}{\beta N_{0}^{2}+h N_{0}}\right)}{\left[\sqrt{\pi\left(\beta N_{0}^{2}+h N_{0}\right)}\right]^{l}}
$$

Thus, the LLF for the $l$ received symbols is given as,

$$
\begin{aligned}
& \Lambda\left(\boldsymbol{r}_{a} ; \boldsymbol{\theta}\right)=-\frac{l}{2} \ln \pi-\frac{l}{2} \ln \left(\beta N_{0}^{2}+h N_{0}\right)-\frac{l \frac{h^{2}}{4}}{\beta N_{0}^{2}+h N_{0}} \\
& -\frac{\sum_{k=1}^{l} r_{a k}^{2}}{\beta N_{0}^{2}+h N_{0}}+\sum_{k=1}^{l} \ln \cosh \left(\frac{h r_{a k}}{\beta N_{0}^{2}+h N_{0}}\right) .
\end{aligned}
$$

Similarly, taking the first-order partial derivative of (19) with respect to $h$ and $N_{0}$ results in

$$
\begin{aligned}
& \frac{\partial \Lambda}{\partial h}=-\frac{\frac{l}{2} N_{0}}{\beta N_{0}^{2}+h N_{0}}-\frac{\frac{l h}{2}\left(\beta N_{0}^{2}+h N_{0}\right)-\frac{l h^{2}}{4}-N_{0} \sum_{k=1}^{l} r_{a k}^{2}}{\left(\beta N_{0}^{2}+h N_{0}\right)^{2}} \\
& +\frac{\beta N_{0}^{2}}{\left(\beta N_{0}^{2}+h N_{0}\right)^{2}} \sum_{k=1}^{l} r_{a k} \tanh \left(\frac{h r_{a k}}{\beta N_{0}^{2}+h N_{0}}\right) \\
& \frac{\partial \Lambda}{\partial N_{0}}=-\frac{\frac{l}{2}\left(2 \beta N_{0}+h\right)}{\beta N_{0}^{2}+h N_{0}}+\frac{\left(2 \beta N_{0}+h\right)\left(\frac{l h^{2}}{4}+\sum_{k=1}^{l} r_{a k}^{2}\right)}{\left(\beta N_{0}^{2}+h N_{0}\right)^{2}} \\
& -\frac{2 \beta N_{0}+h}{\left(\beta N_{0}^{2}+h N_{0}\right)^{2}} \sum_{k=1}^{l} r_{a k} \tanh \left(\frac{h r_{a k}}{\beta N_{0}^{2}+h N_{0}}\right) .
\end{aligned}
$$

Putting the above equations equal to zero and solving them simultaneously gives us following results:

$$
\begin{aligned}
& h^{2}+2 h N_{0}+2 \beta N_{0}^{2}=\frac{4}{l} \sum_{k=1}^{l} r_{a k}^{2}, \\
& h=\frac{2}{l} \sum_{k=1}^{l} r_{a k} \tanh \left(\frac{h r_{a k}}{\beta N_{0}^{2}+h N_{0}}\right) .
\end{aligned}
$$

The above nonlinear equations seem to prohibit the closed form solutions for estimates of $h$ and $N_{0}$. Using the fact, $\tanh (-x)=-\tanh (x)$, the summation term is given by

$\sum_{k=1}^{l} r_{a k} \tanh \left(\frac{h r_{a k}}{\beta N_{0}^{2}+h N_{0}}\right)=\sum_{k=1}^{l}\left|r_{a k}\right| \tanh \left(\frac{h\left|r_{a k}\right|}{\beta N_{0}^{2}+h N_{0}}\right)$

$=\sum_{k=1}^{l}\left|r_{a k}\right| \frac{1-\left[\exp \left(-\left|r_{a k}\right| / N_{0}\right)\right]^{\frac{2 \gamma}{\gamma+\beta}}}{1+\left[\exp \left(-\left|r_{a k}\right| / N_{0}\right)\right]^{\frac{2 \gamma}{\gamma+\beta}}}$

where $\frac{\exp (x)-\exp (-x)}{\exp (x)+\exp (-x)}=\tanh (x)$ is used. It is noticed that for high SNR, where $\left|r_{a k}\right| \gg N_{0}$, the term $\exp \left(-\left|r_{a k}\right| / N_{0}\right)$ is approximated as zero. Thus, the summation term is approximated as

$$
\sum_{k=1}^{l} r_{a k} \tanh \left(\frac{h r_{a k}}{\beta N_{0}^{2}+h N_{0}}\right) \approx \sum_{k=1}^{l}\left|r_{a k}\right| .
$$

Thus, the estimate of $h$ is given as

$$
\widehat{h}=\frac{2}{l} \sum_{k=1}^{l}\left|r_{a k}\right| \text {. }
$$

Using (22) and (26), the estimate of $N_{0}$ is given by

$$
\widehat{N}_{0}=\frac{-\frac{\widehat{h}}{2}+\sqrt{\left(\frac{\widehat{h}}{2}\right)^{2}-\frac{\beta}{2}\left(\widehat{h}^{2}-\frac{4}{l} \sum_{k=1}^{l} r_{a k}^{2}\right)}}{\beta} .
$$

\section{Joint Estimation Using Pilot and Data Symbols}

Assuming independent received symbols, the joint PDF is the product of PDFs resulting from the pilot and data symbols, thus the LLF is given as

$$
\begin{aligned}
& \Lambda\left(\boldsymbol{r}_{a} ; \boldsymbol{\theta}\right)=-\frac{K}{2} \ln \pi-\frac{K}{2} \ln \left(\beta N_{0}^{2}+h N_{0}\right)-\frac{l \frac{h^{2}}{4}+\sum_{k=g+1}^{K} r_{a k}^{2}}{\beta N_{0}^{2}+h N_{0}} \\
& -\frac{\sum_{k=1}^{g}\left(r_{a k}-\frac{c_{m k} h}{2}\right)^{2}}{\beta N_{0}^{2}+h N_{0}}+\sum_{k=g+1}^{K} \ln \cosh \left(\frac{h r_{a k}}{\beta N_{0}^{2}+h N_{0}}\right) .
\end{aligned}
$$

Using similar approximations as the Sec. III-B and taking the first-order partial derivative of (28) and setting them to zero result in the estimates of $h$ and $N_{0}$ as

$$
\begin{aligned}
& \widehat{h}=\frac{2}{K}\left(\sum_{k=1}^{g} c_{m k} r_{a k}+\sum_{k=g+1}^{K}\left|r_{a k}\right|\right), \\
& \widehat{N}_{0}=\frac{-\frac{\widehat{h}}{2}+\sqrt{\left(\frac{\widehat{h}}{2}\right)^{2}-\frac{\beta}{2}\left(\widehat{h}^{2}-\frac{4}{K} \sum_{k=1}^{K} r_{a k}^{2}\right)}}{\beta} .
\end{aligned}
$$

\section{CRAMÉR-RAO LOWER BOUND}

It is noticed that we can have different CRLBs for the different estimators in FM-DCSK system. The completely or fully data-aided (FDA) estimator serves as a bench mark on the variance for all estimators, which is the same as DA but uses all information in the entire packet as training sequence [13][14]. Hence, we derive the CRLB for the FDA estimator. Furthermore, the CRLB for the NDA estimator is also derived.

Since the unknown parameter is a vector, the CRLB for $\mathrm{SNR}$ is given as [16]

$$
C R L B=\frac{\partial g(\boldsymbol{\theta})}{\partial \boldsymbol{\theta}} \boldsymbol{I}^{-1}(\boldsymbol{\theta}) \frac{\partial g(\boldsymbol{\theta})^{T}}{\partial \boldsymbol{\theta}},
$$

where the derivative of the function, $\frac{\partial g(\boldsymbol{\theta})}{\partial \boldsymbol{\theta}}$, is given by

$$
\frac{\partial g(\boldsymbol{\theta})}{\partial \boldsymbol{\theta}}=\left(\frac{1}{N_{0}} \frac{-h}{N_{0}^{2}}\right)
$$

and $\boldsymbol{I}^{-1}(\boldsymbol{\theta})$ is the inverse matrix of the Fisher information matrix (FIM) $\boldsymbol{I}(\boldsymbol{\theta})$, which is given by [16]

$$
\boldsymbol{I}(\boldsymbol{\theta})=\left(\begin{array}{c}
-E_{\boldsymbol{r}_{a}}\left[\frac{\partial^{2} \Lambda\left(\boldsymbol{r}_{a} ; \boldsymbol{\theta}\right)}{\partial h^{2}}\right]-E_{\boldsymbol{r}_{a}}\left[\frac{\partial^{2} \Lambda\left(\boldsymbol{r}_{a} ; \boldsymbol{\theta}\right)}{\partial h \partial N_{0}}\right] \\
-E_{\boldsymbol{r}_{a}}\left[\frac{\partial^{2} \Lambda\left(\boldsymbol{r}_{a} ; \boldsymbol{\theta}\right)}{\partial N_{0} \partial h}\right]-E_{\boldsymbol{r}_{a}}\left[\frac{\partial^{2} \Lambda\left(\boldsymbol{r}_{a} ; \boldsymbol{\theta}\right)}{\partial N_{0}^{2}}\right]
\end{array}\right),
$$

where $E_{\boldsymbol{r}_{a}}\left[f\left(\boldsymbol{r}_{a}\right)\right]=\int_{-\infty}^{+\infty} f\left(\boldsymbol{r}_{a}\right) p\left(\boldsymbol{r}_{a} \mid \boldsymbol{\theta}\right) d \boldsymbol{r}_{a}$. 


\section{A. FDA Estimation}

Using the fact (see [18], pp. 86), i.e. for a Gaussian random variable $X, X \sim N\left(m, \sigma^{2}\right)$, one has

$$
E\left[(X-m)^{n}\right]= \begin{cases}\frac{(2 k) ! \sigma^{2 k}}{2^{k} k !} & n=2 k \\ 0 & n=2 k+1\end{cases}
$$

and taking the second-order partial derivatives of (11) and (12), where $K$ is instead of $g$, with respect to $h$ and $N_{0}$, we can obtain the elements of $\boldsymbol{I}(\boldsymbol{\theta})$. Hence, the FIM of FDA estimator is derived as

$$
\boldsymbol{I}(\boldsymbol{\theta})=\left(\begin{array}{cc}
\frac{K N_{0}\left(\beta N_{0}+h+N_{0}\right)}{2\left(\beta N_{0}^{2}+h N_{0}\right)^{2}} & \frac{2 K \beta N_{0}^{2}+K h N_{0}}{2\left(\beta N_{0}^{2}+h N_{0}\right)^{2}} \\
\frac{2 K \beta N_{0}^{2}+K h N_{0}}{2\left(\beta N_{0}^{2}+h N_{0}\right)^{2}} & \frac{K\left(2 \beta N_{0}+h\right)^{2}}{2\left(\beta N_{0}^{2}+h N_{0}\right)^{2}}
\end{array}\right) .
$$

Furthermore, using (34), (11) and (12), one has $E_{\boldsymbol{r}_{a}}\left[\frac{\partial \Lambda}{\partial h}\right]=$ $E_{\boldsymbol{r}_{a}}\left[\frac{\partial \Lambda}{\partial N_{0}}\right]=0$. It shows that Eq. (10) satisfies the regularity conditions. Thus, according to the Theorem 7.1 in Chapter 7 of [16], this estimator is unbiased.

Substituting (32) and the inverse matrix of (35) into (31), the CRLB for FDA estimator is derived as

$$
C R L B_{F}=\frac{2(\gamma+\beta)^{2}}{K(\gamma+2 \beta)^{2}}\left(\gamma^{2}+4 \gamma+4 \beta\right) .
$$

\section{B. NDA Estimation}

We define the following equations as:

$$
\begin{aligned}
& E_{\boldsymbol{r}_{a}}\left[\sum_{k=1}^{l} r_{a k}^{2}\right]=\frac{l}{2}\left(\beta N_{0}^{2}+h N_{0}+\frac{h^{2}}{2}\right), \\
& E_{\boldsymbol{r}_{a}}\left[\sum_{k=1}^{l} r_{a k} \tanh \left(\frac{h r_{a k}}{\beta N_{0}^{2}+h N_{0}}\right)\right]=\frac{l h}{2}, \\
& E_{\boldsymbol{r}_{a}}\left[\sum_{k=1}^{l} r_{a k}^{2} \cosh ^{-2}\left(\frac{h r_{a k}}{\beta N_{0}^{2}+h N_{0}}\right)\right]=l N_{0}^{2} f(\gamma),
\end{aligned}
$$

where $f(\gamma)=\frac{\exp \left[-\frac{\gamma^{2}}{4(\beta+\gamma)}\right]}{\sqrt{\pi(\beta+\gamma)}} \int_{-\infty}^{+\infty} t^{2} \frac{\exp \left(-\frac{t^{2}}{\beta+\gamma}\right)}{\cosh \left(\frac{t \gamma}{\beta+\gamma}\right)} d t$.

Similarly, taking the second-order partial derivatives of (20) and (21) with respect to $h$ and $N_{0}$, using (37), (38), and (39), we can obtain the elements of $\boldsymbol{I}(\boldsymbol{\theta})$. Hence, the FIM of NDA estimator is derived as

$\boldsymbol{I}(\boldsymbol{\theta})=C\left(\begin{array}{ll}(\gamma+\beta+1)-\frac{2 \beta^{2} f(\gamma)}{(\gamma+\beta)^{2}} & (\gamma+2 \beta)\left(1+\frac{2 \beta \gamma f(\gamma)}{(\gamma+\beta)^{2}}\right) \\ (\gamma+2 \beta)\left(1+\frac{2 \beta \gamma f(\gamma)}{(\gamma+\beta)^{2}}\right) & (\gamma+2 \beta)^{2}\left(1-\frac{2 \gamma^{2} f(\gamma)}{(\gamma+\beta)^{2}}\right)\end{array}\right)$,

where $C=\frac{l}{2 N_{0}^{2}(\gamma+\beta)^{2}}$. Similarly, using (37), (38), (39), (20) and (21), one has $E_{\boldsymbol{r}_{a}}\left[\frac{\partial \Lambda}{\partial h}\right]=E_{\boldsymbol{r}_{a}}\left[\frac{\partial \Lambda}{\partial N_{0}}\right]=0$. Hence, the NDA estimator is also unbiased.

Substituting (32) and the inverse matrix of (40) into (31), the CRLB for NDA estimator is derived as

$$
C R L B_{N}=\frac{2(\gamma+\beta)^{3}\left[(\gamma+\beta)\left(\gamma^{2}+4 \gamma+4 \beta\right)-2 \gamma^{2} f(\gamma)\right]}{l(\gamma+2 \beta)^{2}\left[(\gamma+\beta)^{2}-\left(\gamma^{2}+\gamma+\beta\right) f(\gamma)\right]}
$$

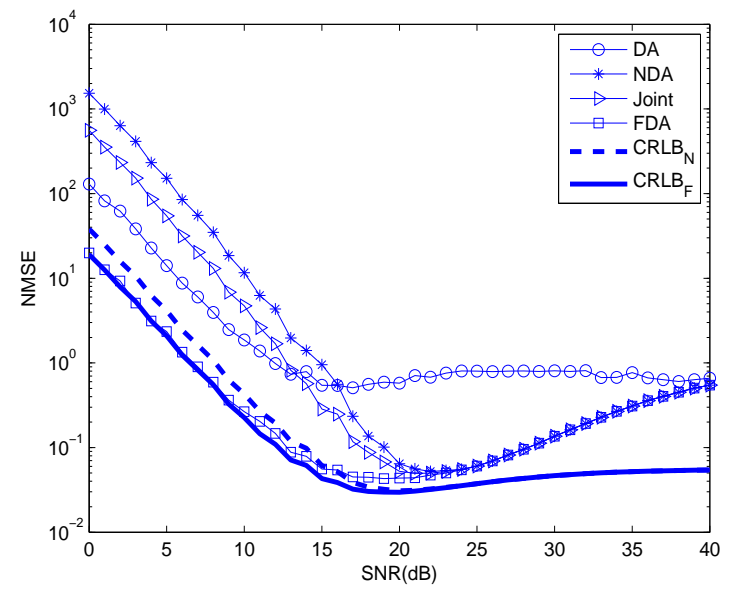

Fig. 2. NMSE vs. true SNR for different proposed estimators, where spreading factor is set to 80 .

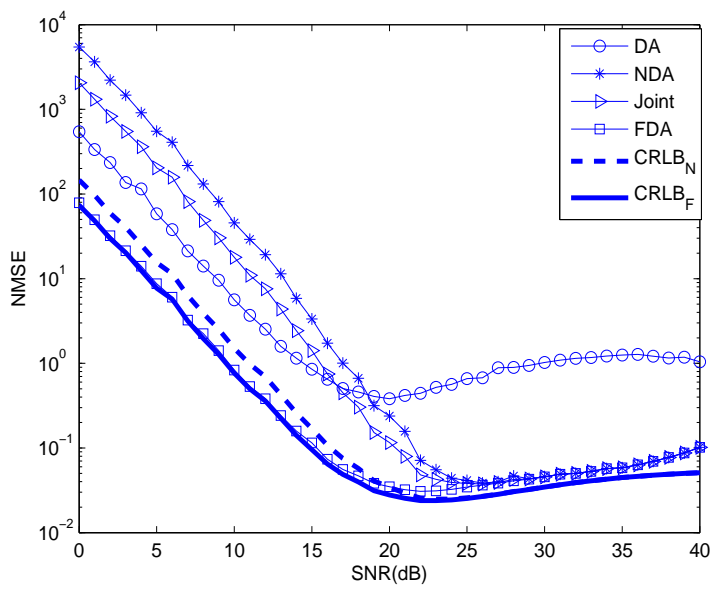

Fig. 3. NMSE vs. true SNR for different proposed estimators, where spreading factor is set to 320 .

For large SNR, $f(\gamma)$ is approximated as zero. Eq. (41) is further approximated as $\frac{2(\gamma+\beta)^{2}}{l(\gamma+2 \beta)^{2}}\left(\gamma^{2}+4 \gamma+4 \beta\right)$, which is the same as Eq. (36) if $l$ equals to $K$.

Note that, the proposed expressions can be applied into DCSK systems, e.g. [5], [6], [9], [18]. At the time, because the term $\beta N_{0}^{2} / 2$ of Eq. (7) is replaced by $\beta N_{0}^{2} / 4$ in DCSK system, $\beta / 2$ is instead of $\beta$ for Eqs. (16), (27), (30), (36) and (41). Hence, the estimation performance for DCSK system is the same as that of FM-DCSK system.

\section{RESULTS AND DISCUSSIONS}

For multipath Rayleigh fading channels, three paths $L=$ 3 are considered having equal average power gain, i.e. $E\left[\left|\alpha_{1}\right|^{2}\right]=E\left[\left|\alpha_{2}\right|^{2}\right]=E\left[\left|\alpha_{3}\right|^{2}\right]=1 / 3$, with the time delays $\tau_{1}=0, \tau_{2}=2$ and $\tau_{3}=5$, respectively. The estimators' performance is evaluated in terms of the normalized mean squared error (NMSE) using simulations and compared to the normalized CRLB defined as:

$$
\operatorname{NMSE}(\widehat{\gamma})=\frac{E\left\{(\gamma-\widehat{\gamma})^{2}\right\}}{\gamma^{2}}, \operatorname{NCRLB}(\gamma)=\frac{C R L B(\gamma)}{\gamma^{2}},
$$




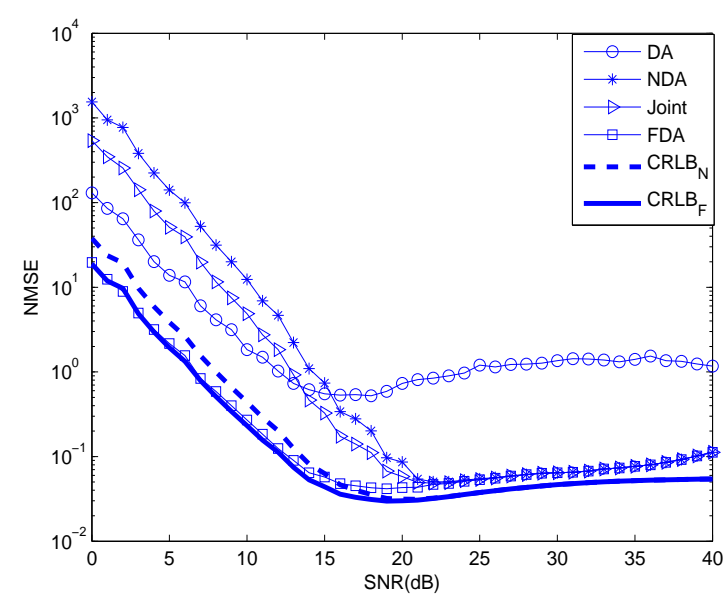

Fig. 4. NMSE vs. true SNR for different proposed estimators without ISI, where spreading factor is set to 80 .

where $\gamma$ is the true value of SNR and $\widehat{\gamma}$ is the estimated value. In simulation, a short packet is considered, comprising 8 pilot symbols and 28 data symbols.

The performance of the proposed estimators is evaluated. Fig. 2 shows the NMSE vs. true SNR for different proposed estimators, where the spreading factor is set to 80 . It is observed that DA estimator outperforms NDA and joint cases in the low SNR region, where it is attributed to the approximations of the nonlinear equations in the section III-B causing the performance loss, while NDA and joint cases outperform DA case in the medium SNR region, because NDA and joint cases use all data of the packet to estimate SNR but DA case only adopts a small amount of pilot data. However, in the high SNR region all estimators perform not well and their NMSEs are the same, because Eq. (4) is non-zero and the system has serious ISI for a small spreading factor, where these two factors also cause the error of channel information estimation resulting in error propagation in noise variance estimation. Here, these factors are referred as to interference term. In a word, the variance of interference term, which is larger than the very small true noise variance at high SNR region, causes inaccurate noise variance estimation.

In order to explain this point, we show the performance of the proposed estimators for a large spreading factor and under no ISI scenario. Fig. 3 plots the NMSE vs. true SNR for different proposed estimators, where the spreading factor is set to 320. Fig. 4 depicts the NMSE vs. true SNR for different proposed estimators without ISI, where the spreading factor is set to 80 . It can be seen that at a large spreading factor the proposed scheme performs well over a wide SNR range, including high SNR region, from Fig. 3, where their performance can achieve the level of no ISI case in Fig. 4. Hence, for a large spreading factor, the variance of interference term can be neglected.

\section{CONCLUSIONS}

In this paper, the ML SNR estimators and CRLBs for SNR estimators serving as a benchmark in FM-DCSK system have been derived under multipath Rayleigh fading channels.
Simulated results show that for a large spreading factor the proposed scheme performs well over a wide SNR range in comparison with CRLBs. The proposed expressions are valid for any binary differential spread spectrum (BDSS)system.

\section{ACKNOWLEDGEMENTS}

This work was supported in part by the National Natural Science Foundation of China under Grant No. 61271241 and the NSERC discovery grant 435243-2013.

\section{REFERENCES}

[1] M. P. Kennedy, G. Kolumban, G. Kis, and Z. Jako, "Performance evaluation of FM-DCSK modulation in multipath environments," IEEE Trans. Circuits Syst.-I, vol. 47, no. 12, pp. 1702-1711, Dec. 2000.

[2] Y. Xia, C. K. Tse, and F. C. M. Lau, "Performance of differential chaos shift-keying digital communication systems over a multipath fading channel with delay spread," IEEE Trans. Circuits Syst.-II, vol. 51, no. 12, pp. 680-684, Dec. 2004.

[3] L. Wang, X. Min, and G. Chen, "Performance of SIMO FM-DCSK UWB system based on chaotic pulse cluster signals," IEEE Trans. Circuits Syst.-I, vol. 58, no. 9, pp. 2259-2268, Apr. 2011.

[4] Y. Fang, P. Chen, and L. Wang, "Performance analysis and optimization of a cooperative FM-DCSK UWB system under indoor environments," IET Networks, vol. 1, no. 2, pp. 58-65, May 2012.

[5] W. Xu, L. Wang, and G. Chen, "Performance of DCSK cooperative communication systems over multipath fading channels," IEEE Trans. Circuits Syst.-I, vol. 50, no. 1, pp. 196-204, Jan. 2011.

[6] Y. Fang, J. Xu, L. Wang, and G. Chen, "Performance of MIMO relay DCSK-CD systems over Nakagami fading channels," IEEE Trans. Circuits Syst.-I, vol. 60, no. 3, pp. 757-767, Mar. 2013.

[7] C. Zhang, L. Wang, and G. Chen, "Promising performance of PA coded SIMO FM-DCSK communication systems," Circuits, Systems and Signal Processing, vol. 27, no. 6, pp. 915-926, Nov. 2008.

[8] Y. Lyu, L. Wang, G. Cai, and G. Chen, "Iterative receiver for M-ary DCSK systems," Accepted by IEEE Trans. Commmun., Apr. 2015.

[9] G. Kaddoum, M. Vu, and F. Gagnon, "Performance analysis of differential chaotic shift keying communications in MIMO systems," in Proc. 2011 IEEE ISCAS, May 2011, pp. 1580-1583.

[10] P. Chen, L. Wang, and F. C. M. Lau, "One analog STBC-DCSK transmission scheme not requiring channel state information," IEEE Trans. Circuits Syst.-I, vol. 60, no. 4, pp. 1-11, Apr. 2012.

[11] N. S. Alagha, "Cramer-Rao bounds of SNR estimates for BPSK and QPSK modulated signals," IEEE Commun. Lett., vol. 5, no. 1, pp. 1012, Jan. 2001.

[12] P. Gao and C. Tepedelenlioglu, "SNR estimation for nonconstant modulus constellations," IEEE Trans. Signal Process., vol. 53, no. 3, pp. 865-870, Mar. 2005.

[13] F. Bellili, R. Meftehi, S. Affes, and A. Stephenne, "Maximum likelihood SNR estimation of linearly-modulated signals over time-varying flatfading SIMO channels," IEEE Trans. Signal Process., vol. 63, no. 2, pp. 441-456, Jan. 2015.

[14] S. A. Hassan and M. A. Ingram, "SNR estimation for M-ARY noncoherent frequency shift keying systems," IEEE Trans. Commun., vol. 59, no. 10, pp. 2786-2795, Oct. 2011.

[15] S. A. Hassan and M. A. Ingram, "SNR estimation in a non-coherent BFSK receiver with a carrier frequency offset," IEEE Trans. Signal Process., vol. 59, no. 7, pp. 3481-3486, July 2011.

[16] S. M. Kay, Fundamentals of Statistical Signal Processing, Estimation Theory. Upper Saddle River, NJ: Prentice Hall, 1993.

[17] L. Wang, G. Cai, G. Chen, "Design and performance analysis of a new multiresolution M-ary differential chaos shift keying communication system," IEEE Trans. Wireless Commun., vol. 14, no. 9, pp. 5197-5208, Sept. 2015.

[18] G. Kaddoum, F. Gagnon, and F.-D. Richardson, "Design and analysis of a multi-carrier differential chaos shift keying communication system," IEEE Trans. Commun., vol. 61, no. 8, pp. 3281-3291, Aug. 2013.

[19] J. G. Proakis, and M. Salehi, Digital Communications, McGraw-Hill, 2007.

[20] A. Abel, W. Schwarz, and M. Gotz, "Noise performance of chaotic communication systems," IEEE Trans. Circuits Syst.-I, vol. 47, no. 12, pp. 1726-1732, Dec. 2000 . 